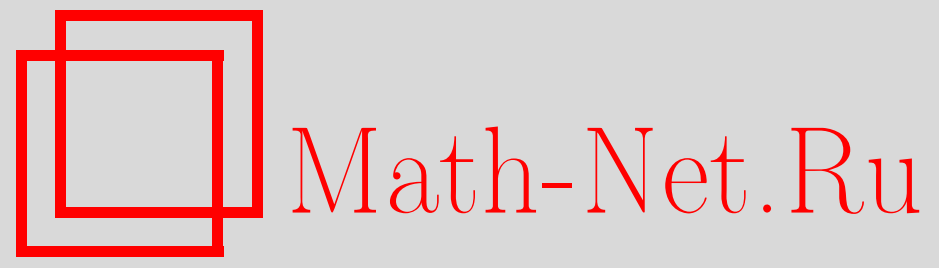

И. В. Орлов, Нормальные разложения операторных пространств над ЛВП, Функи. анализ и его прил., 2002, том 36, выпуск 4, 78-80

DOI: https://doi.org/10.4213/faa223

Использование Общероссийского математического портала MathNet.Ru подразумевает, что вы прочитали и согласны с пользовательским соглашением

http://www . mathnet.ru/rus/agreement

Параметры загрузки:

IP : 54.205 .225 .156

26 апреля 2023 г., 13:30:00

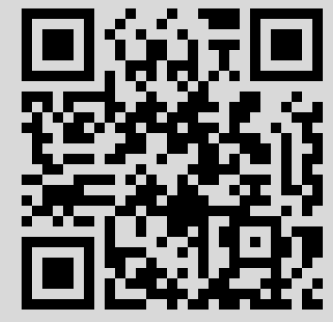


И. В. Орлов

УДК 517.98

\title{
Нормальные разложения операторных пространств над ЛВП
}

\author{
(с) 2002. И. В. Орлов
}

В основе предлагаемого метода лежит понятие нормального индекса линейного оператора, действующего в локально выпуклых пространствах (ЛВП), который характеризует тип непрерывности оператора в терминах индексов соответствующих преднорм. Группировка операторов по их нормальным индексам приводит к нормальным разложениям операторных пространств в индуктивные шкалы ЛВП. Нормальные индексы линейных непрерывных функционалов были введены в работах [1-3] и подробно изучены в [4]. Общие свойства нормальных индексов отображений ЛВП рассмотрены в [5].

Всюду далее $\mathbb{K}$ - поле скаляров, а $E, E_{i}, F, F^{j}$ - ЛВП с соответствующими определяющими системами преднорм $\left\{\|\cdot\|_{s}\right\}_{s \in S},\left\{\|\cdot\|_{s_{i}}\right\}_{s_{i} \in S_{i}},\left\{\|\cdot\|^{t}\right\}_{t \in T}$, $\left\{\|\cdot\|^{t_{j}}\right\}_{t_{j} \in T_{j}}$, индуктивно упорядоченными в соответствии с возрастанием преднорм. Через $(E ; F)$ обозначено пространство линейных непрерывных операторов, действующих из $E$ в $F$, а через $\left(E_{1}, E_{2} ; F\right)$ - пространство билинейных непрерывных операторов, действующих из $E_{1} \times E_{2}$ в $F$.

ОПреДЕЛЕНИЕ 1 . Пусть $A \in(E ; F)$. Для любого $t \in T$ положим

$$
n_{A}(t)=\left\{s \in S:\|A\|_{s}^{t}:=\sup _{\|x\|_{s} \leqslant 1}\|A x\|^{t}<+\infty\right\} .
$$

Многозначное отображение $n_{A}$ назовем нормальным индексом оператора $A$.

ЗАмечАниЕ 2 . Пусть $\operatorname{ray}(S)-$ множество всех лучей в $S$, т. е. таких подмножеств $S$, которые вместе с каждым своим элементом содержат все следующие за ним элементы из $S$. Легко видеть, что $n_{A}$ является отображением из $T$ в $\operatorname{ray}(S)$. Непосредственно проверяется, что множество $\operatorname{ray}(S)$ индуктивно упорядочено отношением $S^{\prime} \preccurlyeq S^{\prime \prime}$, определяемым включением $S^{\prime} \supset S^{\prime \prime}$, и образует решетку; при этом $n_{A}$ является возрастающим отображением. Множество всех нормальных индексов операторов из $(E ; F)$ обозначим через $\mathscr{N}(E, F)$.

ПредЛОЖениЕ 3. Справедливы следующие соотношения:

$$
\begin{gathered}
n_{\sum_{i=1}^{k} c_{i} A_{i}}(t) \preccurlyeq \bigvee_{i=1}^{k} n_{A_{i}}(t), \quad n_{\left(A_{1}, \ldots, A_{k}\right)}\left(t_{1}, \ldots, t_{k}\right)=\bigvee_{i=1}^{k} n_{A_{i}}\left(t_{i}\right), \\
n_{\bigoplus_{i=1}^{k} A_{i}}(t)=\prod_{i=1}^{k} n_{A_{i}}(t), \quad n_{A_{2} \circ A_{1}} \preccurlyeq n_{A_{1}} \circ n_{A_{2}} .
\end{gathered}
$$

Здесь соответственно

$$
\left(A_{1}, \ldots, A_{k}\right): E \rightarrow \prod_{j=1}^{k} F^{j}, \quad \bigoplus_{i=1}^{k} A_{i}: \bigoplus_{i=1}^{k} E_{i} \rightarrow F \quad \text { и } \quad A_{2} \circ A_{1}: E \rightarrow G
$$

(где $\left.A_{1}: E \rightarrow F, A_{2}: F \rightarrow G\right)$.

ОПрЕДЕлЕНИЕ 4. Система локально выпуклых пространств $\vec{F}=\left\{F_{q}\right\}_{q \in Q}$, индуктивно упорядоченная в соответствии с непрерывными вложениями, т. е. 
$q_{1} \preccurlyeq q_{2}$ тогда и только тогда, когда $F_{q_{1}} \subseteq F_{q_{2}}$, называется индуктивной шкалой ЛВП. Сходимость в шкале есть сходимость в каком-либо из пространств шкалы. Дальнейшие определения см. в [6].

ОПрЕДЕЛЕНИЕ 5. Для всякого нормального индекса $n \in \mathscr{N}(E, F)$ рассмотрим векторное пространство $(E ; F)_{n}=\left\{A \in(E ; F): n_{A} \preccurlyeq n\right\}$. Введем в каждом $(E ; F)_{n}$ топологию $\tau_{n}$ как проективную относительно топологий $\tau_{n}(t)$ с определяющими системами норм $\left\{\|\cdot\|_{s}^{t}\right\}_{s \in n(t)}$, заданных формулой (1). Индуктивную шкалу ЛВП $\overrightarrow{(E ; F)}:=\left\{\left((E ; F)_{n}, \tau_{n}\right)\right\}_{n \in \mathscr{N}(E, F)}$ назовем нормальным разложением пространства $(E ; F)$.

Отметим, что нормальные разложения операторных пространств обеспечивают непрерывность отображений вычисления и композиции. В небанаховом случае этого нельзя добиться [7] с помощью единой топологии в операторном пространстве.

Теорема 6. Отображение вычисления $(A, x) \mapsto A x$ непрерывно как отображение $\overrightarrow{(E ; F)} \times E \rightarrow F$ по совокупности переменных. При этом, если $\overrightarrow{(E ; F)}$ - некоторое разложение пространства $(E ; F)$ в индуктивную шкалу ЛВП, при котором отображение вычисления $\overrightarrow{(E ; F)}_{1} \times E \rightarrow F$ непрерывно, то имеет место непрерывное вложение $\overrightarrow{(E ; F)})_{1} \subseteq \overrightarrow{(E ; F)}$.

Теорема 7. Отображение композиции $\left(A_{1}, A_{2}\right) \mapsto A_{2} \circ A_{1}$ непрерывно как отображение $\overrightarrow{(E ; F)} \times \overrightarrow{(F ; G)} \rightarrow \overrightarrow{(E ; G)}$ по совокупности переменных. При этом, если $\overrightarrow{(F ; G)_{1}}$ - некоторое разложение пространства $(F ; G)$ в индуктивную икалу ЛВП, при котором отображение композиции $\overrightarrow{(E ; F)} \times \overrightarrow{(F ; G)} \rightarrow \overrightarrow{(E ; G)}$ непрерывно, то имеет место непрерывное вложение $\overrightarrow{(F ; G)}, \subseteq(\overrightarrow{(F ; G)}$.

ЗАмЕчАНИЕ 8 (гладкая замена переменных в отображениях ЛВП). Примем для отображений $f: E \rightarrow F$ произвольное определение дифференцируемости типа $f(x+h)-f(x)=f^{\prime}(x) h+\varphi(h), f^{\prime}(x) \in(E ; F)$, при условии, что

$$
\frac{\varphi(\lambda h+o(\lambda))}{\lambda} \rightarrow 0 \quad \text { при } \lambda \rightarrow 0 \quad \forall h \in E
$$

для остаточного члена $\varphi(h)$. Отметим, что условие $(2)$ весьма слабое и выполняется практически для всех известных определений дифференцируемости (см. $[8,9])$. Допустим, что отображение $g: \mathbb{R} \rightarrow E$ непрерывно дифференцируемо в точке $\lambda_{0} \in \mathbb{R}$, отображение $f: E \rightarrow F$ дифференцируемо в некоторой окрестности точки $x_{0}=g\left(\lambda_{0}\right) \in E$ и производное отображение $f^{\prime}$ непрерывно в точке $x_{0}$ как отображение в шкалу $\overrightarrow{(E ; F)}$. Тогда отображение $f \circ g: \mathbb{R} \rightarrow F$ также непрерывно дифференцируемо в точке $\lambda_{0}$.

Далее, нормальные разложения операторных пространств позволяют перенести на случай операторов в ЛВП канонические изоморфизмы, широко применяемые в банаховых пространствах.

ТЕОРемА 9. Для любых ЛВП $E_{1}, \ldots, E_{k} u F^{1}, \ldots, F^{m}$ имеют место изометрические изоморфизмы

$$
\overrightarrow{\left(\bigoplus_{i=1}^{k} E_{i} ; F\right)} \cong \prod_{i=1}^{k} \overrightarrow{\left(E_{i} ; F\right)}, \quad \overrightarrow{\left(E ; \prod_{j=1}^{m} F^{j}\right)} \cong \prod_{j=1}^{m} \overrightarrow{\left(E ; F^{j}\right)} .
$$


Поясним, что под изометрическим изоморфизмом ЛВП в соответствующих шкалах здесь понимаются равенства подходящих преднорм в определяющих системах. При этом в прямой сумме $\bigoplus_{i=1}^{k} E_{i}$ мы рассматриваем преднормы $\|\cdot\|_{s_{1}}+$ $\cdots+\|\cdot\|_{s_{k}}$, в прямом произведении $\prod_{j=1}^{m} F^{j}-$ преднормы $\max \left(\|\cdot\|^{t_{1}}, \ldots,\|\cdot\|^{t_{m}}\right)$.

Аналогичным образом, нормальные индексы билинейных непрерывных операторов приводят к изоморфизмам нормальных разложений соответствующих операторных пространств. В частности, канонический изоморфизм между линейными и билинейными формами принимает вид $\overrightarrow{\left(E_{1} ; \overrightarrow{E_{2}^{*}}\right)} \cong \overrightarrow{\left(E_{1}, E_{2}\right)^{*}}$. Отметим, что «функциональные» операторы $A: E_{1} \rightarrow E_{2}^{*}$ находят широкое применение в теории управления [10].

\title{
ЛИТЕРАТУРА
}

1. Orlov I. V. In: Oper. Theory Adv. Appl., Vol. 118, Birkhäuser, Basel, 2000, pp. 321333. 2. Orlov I. V. In: Spectral and Evolution Problems, Vol. 10 (Proc. of the Tenth Crimean Autumn Math. School), Natl. Taurida Univ. "V. Vernadsky", Simferopol, 2000, pp. 35-39. 3. Орлов И. В. Динамические системы, 16, 165-171 (2000). 4. Orlov I. V. Methods of Functional Analysis and Topology, 8, No. 3, 61-71 (2002). 5. Орлов И. В. In: Spectral and Evolution Problems, Vol. 11 (Proc. of the Eleventh Crimean Autumn Math. School), Natl. Taurida Univ. "V. Vernadsky", Simferopol, 2001, pp. 18-29. 6. Волевич Л. Р., Гиндикин С. Г. Обобщенные функции и уравнения в свертках. Наука, M., 1994. 7. Köthe G. Topologishe lineare Räume. Springer-Verlag, Berlin, 1960. 8. Балабанов B. A. Некоторые вопросы нелинейного функционального анализа и их приложения. Мецниереба, Тбилиси, 1982. 9. Сухинин М. Ф. Избранные главы нелинейного анализа. Изд-во Росс. ун-та дружбы народов, М., 1992. 10. Зәуровский M. З., Мельник B. C. Нелинейный анализ и управление бесконечномерными системами. Наукова думка, Киев, 1999.

Таврический национальный университет,

Симферополь, Украина

Поступило в редакцию e-mail: old@tnu.crimea.ua

31 октября 2001 г.

УДК 515.164.174

\section{О функциях, все критические точки которых содержатся в шаре}

\author{
(c) 2002. П. Е. ПушкАРь
}

Настоящая заметка дает ответ на следующий вопрос В. И. Арнольда ([1, задача 1993-45]). Рассмотрим на связном замкнутом многообразии функцию с конечным числом критических точек. Подходящим диффеоморфизмом можно перевести эту функцию в функцию, все критические точки которой содержатся в некотором (малом) открытом шаре. Рассмотрим ограничение построенной функции на шар. Для каких пар связных компактных многообразий без края $M_{1}^{n}$, $M_{2}^{n}$ найдутся функции, совпадающие на шарах, содержащих все их критические точки? Ответ дает следующая теорема:

ТеоремА. Такие функции существуют, если и только если эйлеровы характеристики многообразий $M_{1}^{n}$ и $M_{2}^{n}$ pавнь, $\chi\left(M_{1}^{n}\right)=\chi\left(M_{2}^{n}\right)$.

* Работа частично поддержана Российским фондом фундаментальных исследований (проект 0201-00655). 